\title{
JUURNAL.RU
}

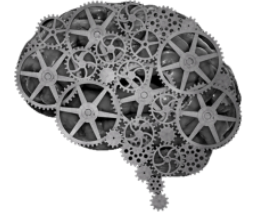

COMPANY GROUP "INTELLEKT"

\author{
Дергунова Е.Е., Тимофеев А.В., Козлов В.В. \\ Самарский государственный архитектурно-строительный университет \\ Самара, Россия
}

doi: 10.18411/lj2016-5-2-09

\section{Разработка программного обеспечения информационных систем при помощи CASE-средств}

Ключевые слова: CASE, программное обеспечение,информационные системы, база данных, разработка, технологии.

За последнее десятилетие сформировалось новое направление в программотехнике - CASE (Computer-AidedSoftware/SystemEngineering) разработка программного обеспечения информационных систем при поддержке компьютера.

Под термином CASE-средства понимаются программные средства, поддерживающие процессы создания и сопровождения ИС, включая анализ и формулировку требований, проектирование прикладного ПО и БД, генерацию кода, тестирование, документирование, обеспечение качества, конфигурационное управление и управление проектом, а также другие процессы.

Основная цель CASE состоит в том, чтобы отделить проектирование ПО от его кодирования и последующих этапов разработки, а также скрыть от разработчиков все детали среды разработки и функционирования ПО. При использовании CASE-технологий изменяются все этапы жизненного цикла ПО ИС. Большинство существующих CASE-средств основано на методологиях структурного или объектно-ориентированного анализа и проектирования, 
использующих спецификации в виде диаграмм или текстов для описания внешних требований, связей между моделями системы, динамики поведения системы и архитектуры программных средств. Такие методологии обеспечивают строгое и наглядное описание проектируемой системы, которое начинается с ее общего обзора и затем детализируется, приобретая иерархическую структуру с все большим числом уровней. CASE-технологии успешно применяются для построения практически всех типов систем ПО, однако устойчивое положение они занимают в следующих областях:

- обеспечение разработки делового и коммерческого ПО;

- разработка системного и управляющего ПО;

- Достоинства CASE-средств:

- улучшают качество создаваемого ПО за счет средств автоматического контроля;

- позволяют за короткое время создавать прототип будущей системы, что позволяет на ранних этапах оценить ожидаемый результат;

- ускоряют процесс проектирования и разработки;

• поддерживают развитие и сопровождение разработки;

• поддерживают технологии повторного использования компонента разработки.

Появлению CASE-технологии и CASE-средств предшествовали исследования в области методологии программирования. Программирование обрело черты системного подхода с разработкой и внедрением языков высокого уровня, методов структурного и модульного программирования, языков проектирования и средств их поддержки, формальных и неформальных языков описаний системных требований и спецификаций и т. д. В 70—80-х гг. стала на практике применяться структурная методология, предоставляющая в распоряжение разработчиков строгие формализованные методы описания АИС и принимаемых технических решений. Она основана на наглядной графической технике: для описания 
различного рода моделей АИС используются схемы и диаграммы. Наглядность и строгость средств структурного анализа позволяла разработчикам и будущим пользователям системы с самого начала неформально участвовать в её создании, обсуждать и закреплять понимание основных технических решений. Однако широкое применение этой методологии и следование её рекомендациям при разработке контактных АИС встречалось достаточно редко, поскольку при неавтоматизированной разработке это практически невозможно. Это и способствовало появлению программно-технических средств особого класса CASE-средств, реализующих CASE-технологию создания и сопровождения АИС.

\section{Литература:}

1. Калянов Г.Н.CASE. Структурный системный анализ/ Калянов Г.Н. 1996.

2. Венгров A.M. CASE-современные методы и средства проектирования информационных систем./Венгров А.М. - М.: Финансы и статистика, 1998. 